\title{
Shape and lattice distortion effects on infrared absorption spectra of olivine particles
}

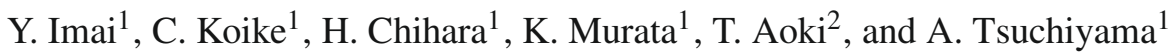 \\ 1 Department of Earth and Space Science, Osaka University, Toyonaka 560-0043, Japan \\ e-mail: imai@astroboy.ess.sci.osaka-u.ac.jp \\ 2 Department of Earth and Planetary Sciences, Faculty of Sciences, Kyushu University, Hakozaki, 812-8581, Japan
}

Received 27 February 2009 / Accepted 18 May 2009

\section{ABSTRACT}

\begin{abstract}
We prepared sub-micron olivine $\left(\mathrm{Fo}_{92}\right)$ particles of different shapes and different degrees of crystal lattice distortion, and studied both effects on the infrared absorption spectra experimentally. We found that the peak positions of the spectra shifted to short wavelength when the number fraction of spherical grains became large, consistent with spectral calculations using the continuous distributions of ellipsoids (CDE) model. We also found for the first time that the spectral features become broader by crystal lattice distortion. The peak intensities are affected by both effects. The peak intensity decreases with lattice distortion in general, but some peaks are largely affected by particle shape. The spectral difference of olivine measured in different laboratories can be explained by both the shape and lattice distortion effects through sample preparation methods. Broadening features in the infrared spectra of forsterite ejecta from comet Tempel 1 obtained in the Deep Impact mission, and forsterite crystals in circumstellar regions, suggest that forsterite crystals undergo lattice distortion.
\end{abstract}

Key words. stars: circumstellar matter - infrared: ISM - ISM: dust, extinction - methods: laboratory

\section{Introduction}

Circumstellar and interstellar dust has been investigated by comparing infrared spectra of astronomical observations and laboratory measurements. Crystalline silicates (e.g., olivine $\left((\mathrm{Mg}, \mathrm{Fe})_{2} \mathrm{SiO}_{4}\right)$, and pyroxene $\left.\left((\mathrm{Mg}, \mathrm{Fe})_{2} \mathrm{SiO}_{3}\right)\right)$ have been identified in circumstellar environments around oxygen rich young and evolved stars (e.g., Waters et al. 1996; Waelkens et al. 1996). It is known that IR spectra of solid-state material depend not only on intrinsic properties, such as temperature, structure and chemical composition, but also on extrinsic properties related to light-scattering of fine particles, such as particle size, shape, and aggregation (Bohren \& Huffman 1983). In order to understand observational data correctly and infer the physical conditions of circumstellar environments, laboratory spectroscopic studies of these dependencies are required.

In recent years, a considerable number of studies have been conducted on the dependencies of the intrinsic properties. So far, effects of chemical composition and temperature on infrared absorption spectra of silicates have been investigated experimentally. For example, Jäger et al. (1998) reported the peak shift of the spectra of four olivine samples depending on the composition change from $\mathrm{Mg}$ to Fe end members. Chihara et al. (2002) and Koike et al. (2003) clarified the compositional dependency of pyroxene and olivine IR spectra. Bowey et al. (2001) measured the temperature effects on IR spectra of olivine and pyroxene. Koike et al. (2006) revealed the temperature dependence of IR spectra of olivine, and estimated temperatures in circumstellar environments. Fabian et al. (2000) and Murata et al. (2007) reported the evolution of IR spectra by crystallization of amorphous silicates.

In contrast, the extrinsic properties related to light-scattering of fine particles have not been determined experimentally yet, because, in general, it is difficult to prepare fine particles of less than $1 \mu \mathrm{m}$, while still controlling the properties related to light-scattering, such as shape and size. Since the size of dust is considered to be sub-micron, which is smaller than the wavelength of infrared radiation, scattering from the dust is through Rayleigh scattering. Thus, measurements of infrared absorption spectra in the laboratory also have been carried out in the regime of Rayleigh scattering. The work of Tamanai et al. (2006) is an experimental study of the dependency of the extrinsic properties of IR spectra. Using aerosol techniques, particles were well dispersed in air, and the structures of the particles can be analyzed by in situ microscopic imaging. They investigated the influence of the medium and of particle morphology on IR spectra. They found that the difference between IR spectra of irregular and ellipsoidal forsterite particles depends on the surrounding medium. However, the irregular and ellipsoidal particles used are commercial products made by different companies. So these particles may have differences not only of shape but also other properties. It is difficult to control the properties of fine particles, and it is possible that the difficulty gives rise to differences of IR spectra, when IR spectra are measured in the laboratory. In principle, there are significant differences in infrared spectra measured in different laboratories. This is a crucial problem for comparison between observation and laboratory data. It is inferred that the difference is caused not only by the dependencies of the intrinsic properties but also by the dependency related to light-scattering of fine particles.

An example of the difference is the infrared spectrum of forsterite $\left(\mathrm{Mg}_{2} \mathrm{SiO}_{4}\right)$ particles measured by Koike et al. (1999) (called the Kyoto sample hereafter), and by Jäger et al. (1998) (Jena sample) on peak intensities, positions, and shapes (Molster et al. 2002). The mass absorption coefficients of the Kyoto samples are large as twice as the Jena samples, while the peak widths of the Kyoto samples are narrower than those of the Jena 
samples, especially for the $33 \mu \mathrm{m}$ feature. Molster et al. (2002) proposed that the difference is due to the crystallinity of the measured samples; that is, the Kyoto sample was a single crystal made by the Czochralsky method (Koike et al. 2000), while the Jena sample was crystallized from a melt by cooling and is probably polycrystalline (Jäger et al. 1998). On the other hand, Koike et al. (2006) proposed that the difference is not caused by crystallinity but particle shape. They pointed out that the positions of many peaks of the Jena sample are located at shorter wavelength than the Kyoto sample. The difference is about $0.05-0.1 \mu \mathrm{m}$ for $10,11.2$, and $19.5 \mu \mathrm{m}$ peaks, and about $0.5 \mu \mathrm{m}$ for 23 and $33 \mu \mathrm{m}$ peaks. They suggested that the particle shape of the Jena sample is more spherical than the Kyoto sample, because the peak positions for olivine particles with near-spherical shape are located at shorter wavelengths than those with elongated or flat ellipsoidal shapes, as pointed out by Fabian et al. (2001).

In this paper, we propose a hypothesis that the spectrum difference is caused by differences in sample preparation; the Kyoto sample was prepared by grinding in a mortar by hand, and the particle shapes are irregular, while the Jena sample was prepared by grinding mechanically using a ball mill, and the particle shapes are spherical. Milling in a ball mill is indeed used as a technique for amorphization of organic matter (Font et al. 1997). It is possible that the crystal lattice of the Jena sample is distorted due to the strong impacts of the ball mill grinding. The purpose of this study is to test this hypothesis and to investigate the dependence of particle shape and lattice distortion on infrared absorption spectra of olivine particles. For this, we prepared fine particles from one natural olivine by different grinding methods to control their shape, and annealed them to relax lattice distortion. Then, we examined the variation in infrared absorption spectra to evaluate the effects of the particle shape and lattice distortion. Finally, we discuss differences in infrared spectra from different laboratories.

\section{Experiment}

We prepared sub-micron particles of olivine (Fo92: from San Carlos, Arizona, USA) by grinding in a mortar by hand and a planetary ball mill. Since the Fe-bearing olivine sample is softer than the $\mathrm{Mg}$ end-member (forsterite), it is more suitable to examine the change of shape and lattice distortion by these sample preparation methods. Olivine crystals were crushed in a tungsten carbide mortar by hand for about $1 \mathrm{~h}$. The samples were ground in an alumina mortar until the grains were less than $1 \mu \mathrm{m}$ in size (we named this sample "hand-milled"). In addition, to obtain smaller grains, these particles were ground in a planetary ball mill using a zirconia pod and balls, with a rotation speed of $350 \mathrm{rpm}$ for 10-20 h (we named this sample "ball-milled"). In this way, we produced very fine grains (of less than $0.5 \mu \mathrm{m}$ in size). This sample may undergo crystal lattice distortion by the collisions in the ball mill. In order to relax the distortion, the sample was annealed at 650 and $1000{ }^{\circ} \mathrm{C}$ for $3 \mathrm{~h}$. To prevent oxidation of the $\mathrm{Fe}^{2+}$ cation, oxidation-reduction conditions in the furnace were controlled on the iron-wüstite buffer (partial pressure of oxygen is $10^{-22.8}$ atm) curve using $\mathrm{H}_{2}$ and $\mathrm{CO}_{2}$ mixed gas (we named this sample "annealed at 650 and $1000{ }^{\circ} \mathrm{C}$ ").

The size and shape of the samples were observed with a field emission scanning electron microscope (FE-SEM, JEOL7001F) at Osaka University and the degree of lattice distortion was obtained by X-ray powder diffraction (XRD) analysis using using a Gandolfi camera at Kyushu University. Conventional powder XRD measurement was performed with chromium $\mathrm{K} \alpha$ radiation $(\lambda=2.2896 \AA)$. The infrared absorption spectra of the
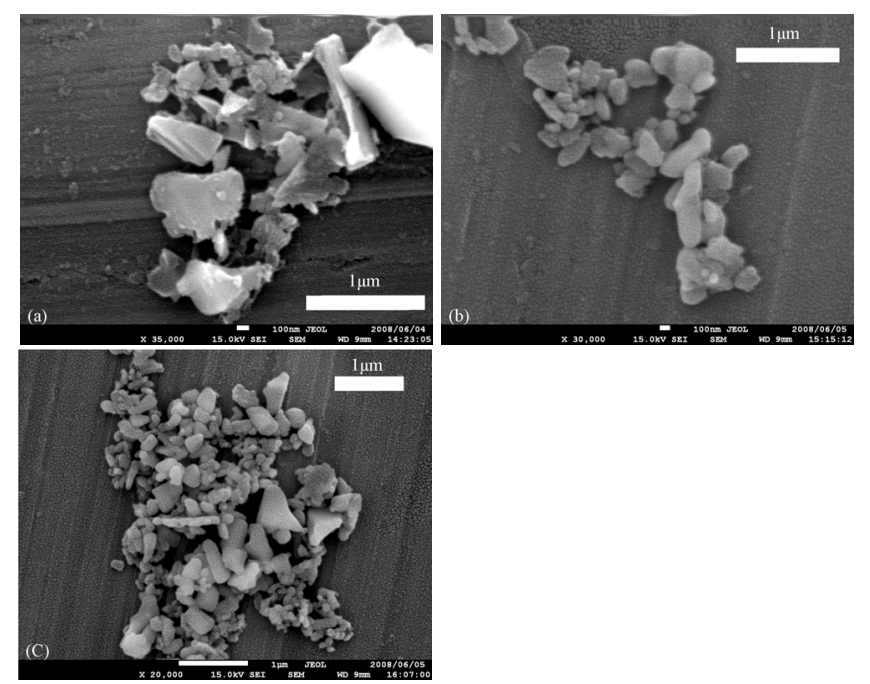

Fig. 1. SEI images of the samples. a) Hand-milled sample. b) Ballmilled sample. c) Annealed sample.

samples were measured by FT-IR spectrometry (Nicolet Nexus 670) at Osaka University. The samples were dispersed and embedded in $\mathrm{KBr}$ and polyethylene for the mid-IR $(1.4-25 \mu \mathrm{m})$ and the far-IR $(15-200 \mu \mathrm{m})$ measurements, respectively. The mass absorption coefficient, $\kappa$ was obtained from the absorbance spectra by use of the following equation;

$\kappa=\frac{S}{M} \ln \left(\frac{1}{T}\right)$

where $S$ is the surface area of the sample pellet, $M$ is the sample mass in the pellet, and $T$ is the transmittance.

\section{Results}

Figure 1 shows particle shapes of "hand-milled", "ball-milled", and "annealed" samples. Before grinding, particles have sharp edges and are irregular in shape (Fig. 1a). The particle size shows variation ranging from $0.2 \mu \mathrm{m}$ to $2 \mu \mathrm{m}$. After grinding (Fig. 1b), the sharp edges have disappeared and the particle shape becomes more spherical with smaller aspect ratios, and particles become roughly uniform (about $0.1-0.5 \mu \mathrm{m}$ ). After annealing, the particle sizes and shapes are similar to those of the ball-milled sample, and the particles are not coarsened by the annealing (Fig. 1c).

The XRD patterns of "hand-milled", "ball-milled", and "annealed" samples are shown in Fig. 2. All peaks in the XRD patterns were assigned to olivine. The samples may be contaminated by material from the ball mill pod, balls and mortar during ball milling. However the XRD profiles did not show any peaks of zirconia. The XRD peaks of hand-milled sample were very sharp, showing good crystalline quality. On the other hand, after grinding by the ball mill, XRD peaks became broad and the intensity decreased. This change was especially remarkable for peaks at large angles. The XRD peaks became broader for longer durations. The change of the XRD patterns corresponds to lattice distortion of olivine crystals by impact during grinding in the ball mill. The XRD peaks of annealed samples became narrower as the annealing temperature increased. This result shows that the lattice distortions were relaxed by the annealing. We evaluated the degree of the lattice distortion $e$ from the XRD patterns, whose variation of lattice spacing corresponds to (Wilson 1962)

$\beta=4 e \tan \theta$ 


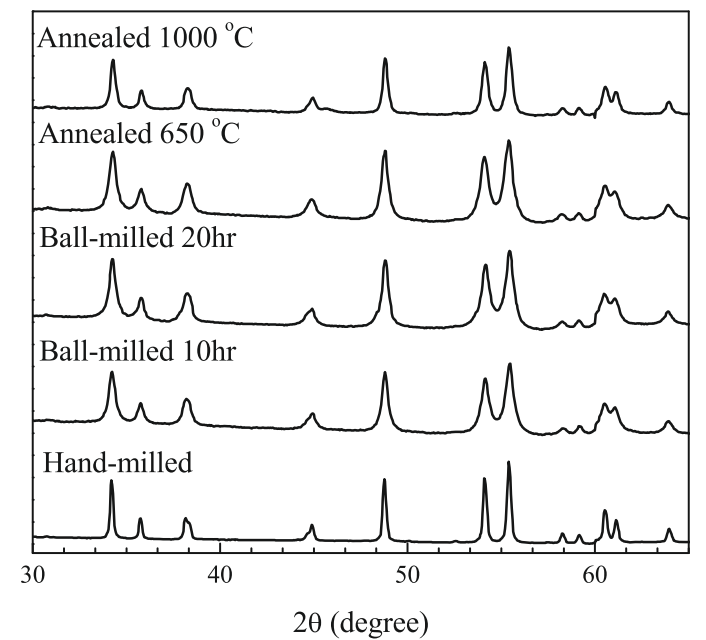

Fig. 2. XRD patterns of each sample. The X-ray wavelength was $2.2896 \AA$.

where $\beta$ is the full width of half maximum (FWHM) of each $\mathrm{XRD}$ peak, $\theta$ is degree of each peak position. However, the widths of the XRD peak are dependent not only on lattice distortion but also other effects such as instrumental error, and crystallite size. So, we added the constant $C$ to the equation, in order to describe these dependencies.

$\beta=4 e \tan \theta+C$.

Strictly, $\mathrm{C}$ has an angular dependency. However, it is not useful to estimate trends in the slopes of the fitting lines. For example, the size dependence is much weaker than that of lattice distortion in this experiment. The spread of the FWHM of the crystalline size dependency was evaluated by (Scherrer 1918)

$\beta_{\text {size }}=\frac{K \lambda}{D \cos \theta}$

where $K$ is the Scherrer constant, $\lambda$ is the wavelength of the $\mathrm{X}$-ray, and $D$ is the crystalline size. In this experiment, the wavelength of X-ray is $2.289 \AA$, and the grain size is about $100 \mathrm{~nm}$. Although $K$ depends on crystalline shape, the value used is approximately $0.5-1.5$. Therefore, the value of $\frac{K \lambda}{D}$ is about $10^{-2}$. When $\theta$ is large, $\cos \theta$ becomes small, and $\beta_{\text {size }}$ becomes large. However, the spread of $F W H M$ by lattice distortion also becomes large when $\theta$ is large. For example, using Eq. (4), the values of $\beta_{\text {size }}$ are about 0.01 and 0.04 when $\theta$ is 30 and $75(\tan \theta$ is about 0.6 and 3.5). The values of $\beta_{\text {size }}$ are smaller than the values of $\beta$.

Figure 3 shows the relation between $\beta$ and $\tan \theta$. By Eq. (3), the $e$ value of each sample is calculated from the relation between gradient of the $\beta$ and $\tan \theta$. The values of lattice distortion $e$, for samples hand-milled, ball-milled for $20 \mathrm{~h}$, and annealed at $1000{ }^{\circ} \mathrm{C}$ were $0.043,0.213$, and $0.111 \%$, respectively. This result shows that lattice distortion occurred from ball milling, and was partially relaxed by the annealing.

The infrared absorption spectra of "hand-milled", "ballmilled for $20 \mathrm{hr}$ ", and "annealed at $1000^{\circ} \mathrm{C}$ " samples are shown in Fig. 4. Table 1 shows peak positions and FWHMs for each spectrum. The spectra are similar to a spectrum of San Carlos olivine measured by Koike et al. (2006), and did not show any peaks of contamination, such as material from the ball mill pod, balls and mortar.

Many peaks of the spectrum of the ball-milled sample shifted to shorter wavelength compared to the hand-milled sample. For

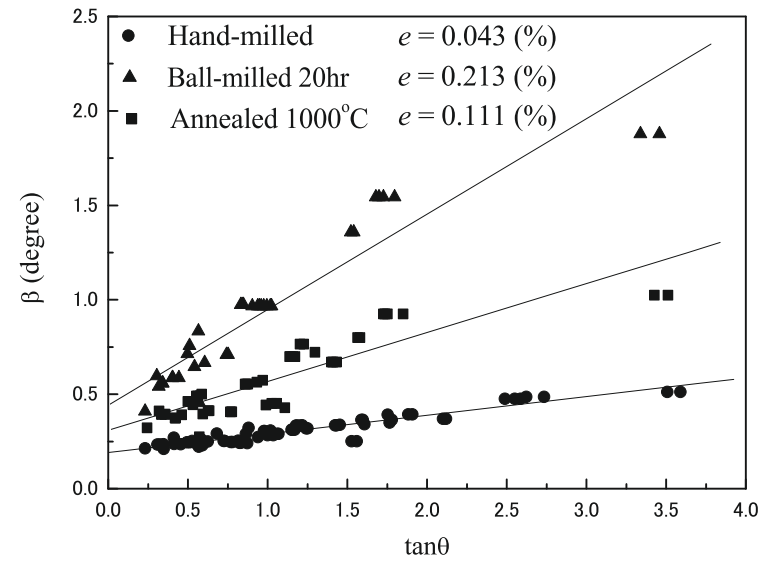

Fig. 3. Relations between $F W H M$ of XRD peaks of each sample and lattice distortion. $\beta$ is $F W H M$ of each XRD peak, and $e$ is lattice distortion.

example, the peak positions of the 24 and $34 \mu \mathrm{m}$ feature of the hand-milled sample shifted from those of the ball-milled by 0.25 and $0.33 \mu \mathrm{m}$, respectively. Peaks of the spectrum of the annealed sample also are at shorter wavelength than the hand-milled sample and are similar to those of the ball-milled sample. In terms of shape of spectral features, the spectrum of the ball-milled sample became broader than the hand milled sample, and the peak intensities of the ball-milled sample were generally lower than the hand-milled sample. This broadening was particularly remarkable in the long wavelength region above $13 \mu \mathrm{m}$. For example, the $F W H M$ of the $16 \mu \mathrm{m}$ feature changed from 0.81 to $1.12 \mu \mathrm{m}$ and that of the $34 \mu \mathrm{m}$ feature from 2.66 to $2.92 \mu \mathrm{m}$ for the hand-milled and ball-milled samples, respectively. However, the spectral features of the annealed sample were as sharp as the hand-milled sample, and the peak intensity of the annealed sample was similar to that of the hand-milled sample. In addition, the shapes of $19 \mu \mathrm{m}$ feature are different among the three samples. This feature includes two peaks at about 18.5 and $19.5 \mu \mathrm{m}$, and the $19 \mu \mathrm{m}$ feature is sensitive to the ball-milling and annealing. The continuum absorptions of the samples also differ. It is surprising that the continuum of the annealed sample is lower than that of the hand-milled sample. It may be related to its crystallinity, however the reason is not known.

\section{Discussion}

\subsection{Lattice distortion effect on infrared spectra}

Table 2 shows a summary of the samples with particle shapes and lattice distortions determined by the SEM observation and the XRD measurements. In order to discuss the lattice distortion effect on infrared spectra, we compared the spectrum of two different samples whose shapes are similar but whose lattice distortions are different. Therefore, we used "ball-milled" and "annealed" samples (Table 2). The peak positions are similar for both spectra, while the spectral peaks of the ball-milled sample with large lattice distortions are broader than the annealed sample, especially for the longer wavelength region of greater than about $13 \mu \mathrm{m}$ (Fig. 4). The peak intensity of the ball-milled sample is generally lower than the annealed sample, although there are variations (the differences are small for the 10, 11.5 and $16 \mu \mathrm{m}$ features, while larger for other features). As a result, we found that the crystal lattice distortion affects the peak broadening in infrared spectra. Peak intensity is also affected for some peaks. 
Table 1. Peak positions of each band for each samples (in $\mu \mathrm{m}$ ).

\begin{tabular}{ccccccc}
\hline \hline & Hand-milled & $F W H M$ & Ball-milled & $F W H M$ & Annealed & $F W H M$ \\
\hline $10.1 \mu \mathrm{m}$ & 10.11 & & 10.06 & & 10.03 & \\
$10.4 \mu \mathrm{m}$ & 10.47 & & 10.46 & & 10.46 & \\
$11.3 \mu \mathrm{m}$ & 11.30 & & 11.25 & & 11.29 & \\
$12 \mu \mathrm{m}$ & 11.91 & & 11.89 & & 11.91 & \\
$16 \mu \mathrm{m}$ & 16.45 & 0.81 & 16.44 & 1.12 & 16.38 & 0.66 \\
$20 \mu \mathrm{m}$ & 19.79 & & 19.59 & & 19.55 & \\
$24 \mu \mathrm{m}$ & 24.15 & 2.46 & 23.90 & 2.61 & 23.92 & 2.33 \\
$26 \mu \mathrm{m}$ & 26.46 & & 26.39 & & 26.39 & \\
$27 \mu \mathrm{m}$ & 27.73 & & 27.58 & & 27.66 & \\
$34 \mu \mathrm{m}$ & 34.06 & 2.66 & 33.73 & 2.92 & 33.84 & 2.72 \\
$50 \mu \mathrm{m}$ & 50.5 & & 50.3 & & 50.4 & \\
$70 \mu \mathrm{m}$ & 73.2 & 2.1 & 72.6 & 3.6 & 72.5 & 2.5 \\
\hline
\end{tabular}
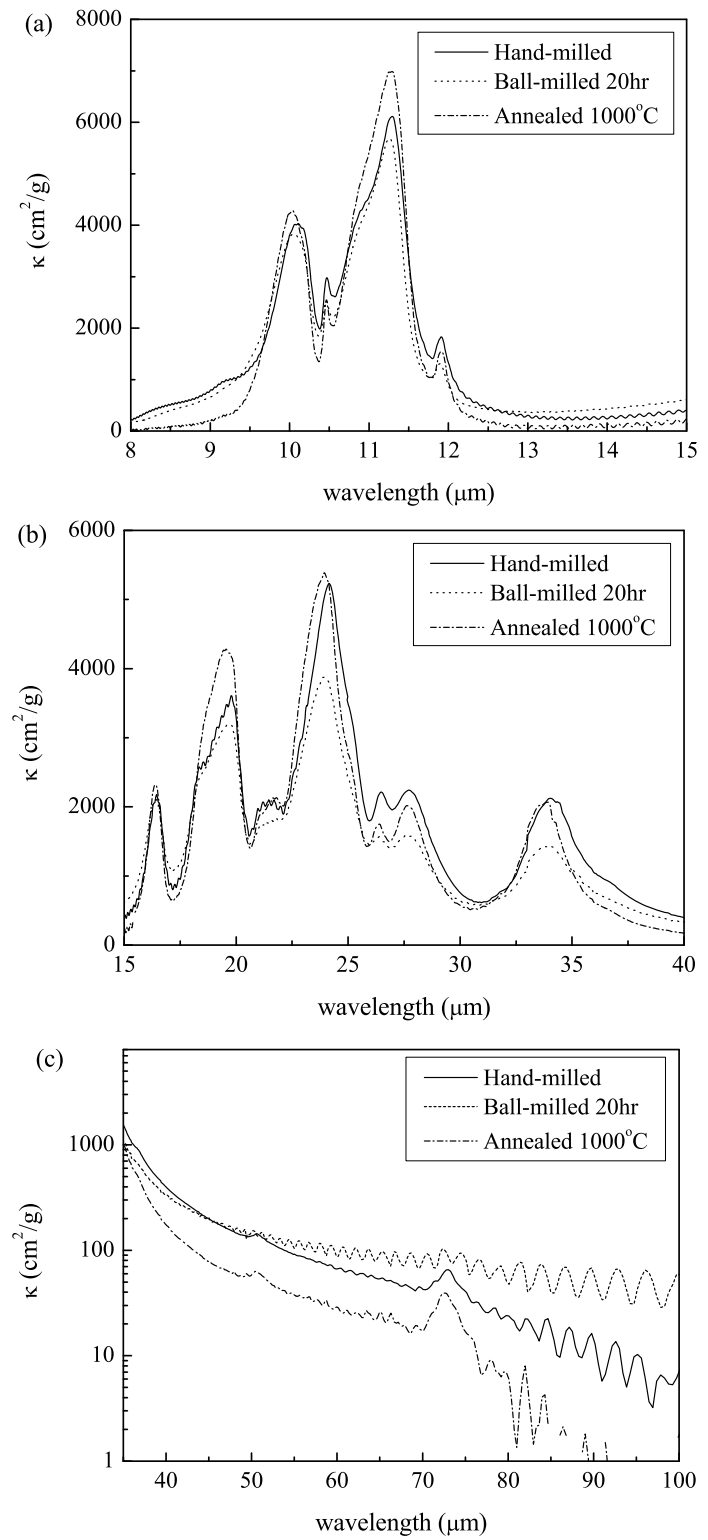

Fig. 4. The IR spectra of different shape and lattice distortion samples a) from 8 to $15 \mu \mathrm{m}$, b) from 15 to $40 \mu \mathrm{m}$, and c) 35 to $100 \mu \mathrm{m}$.

\subsection{Shape effect on spectra}

In order to discuss shape effects on infrared spectra, we compared the spectrum of two different samples whose shapes
Table 2. Shape and lattice distortion of each sample.

\begin{tabular}{ccc}
\hline \hline & Shape & Lattice distortion \\
\hline Hand-milled & Irregular with sharp edges & Very small \\
Ball-milled & Rounded & Large \\
Annealed & Rounded & Small (relaxed) \\
\hline
\end{tabular}

are different but whose lattice distortions are similar. We used the spectra of the "hand-milled" sample with irregular particle shapes and the "annealed" sample with rounded shapes (Table 2). Many peak positions (e.g., 10.1, 11.3, 24 and $34 \mu \mathrm{m}$ ) in the spectrum of the annealed sample are at shorter wavelengths than the hand-milled sample (Fig. 4). In general, the peak intensities of the annealed sample are slightly higher than those of the hand-milled sample. It also should be noted that the shapes of the $19 \mu \mathrm{m}$ feature are different between the two samples. Therefore, we conclude that particle shape mainly affects the peak position. The peak intensities also are slightly affected by the shape.

The shape effect can be discussed by comparing the experimental results with those calculated. Fabian et al. (2001) showed calculated IR spectra of spheres, and two continuous distributions of ellipsoids (CDEs) of forsterite and olivine (Fo95). They considered the CDEs with random orientations, and the distributions are characterized either by equal probability of all shapes (CDE1; Bohren \& Human, 1983), or by a weighting where nearspherical shapes are most probable (CDE2; Ossenkopf et al. 1992). In both calculated spectra of forsterite and olivine, the spectral peak positions of the near spherical particles (CDE2) shift to shorter wavelength than the more elongated and flat ellipsoidal shapes (CDE1). The trend in the shift of peak positions is not different between the spectra of forsterite and olivine. This is consistent with our experimental result, although the composition of the samples is different.

\subsection{Difference in spectra by different laboratories}

As already mentioned, the IR spectra of a mineral measured in different laboratories are different from each other, and this poses serious problems in estimating the properties of circumstellar dust by comparing astronomical observations and laboratory data. Figure 5 shows IR spectra of San Carlos Olivine measured for the Kyoto and Jena samples. The spectral features of the hand-milled sample are similar to that of the Kyoto sample (Koike et al. 2006), in which the sample was prepared by hand-milling, while that of the ball-milled sample is similar to that of the Jena sample (Fabian et al. 2001), in which the sample 


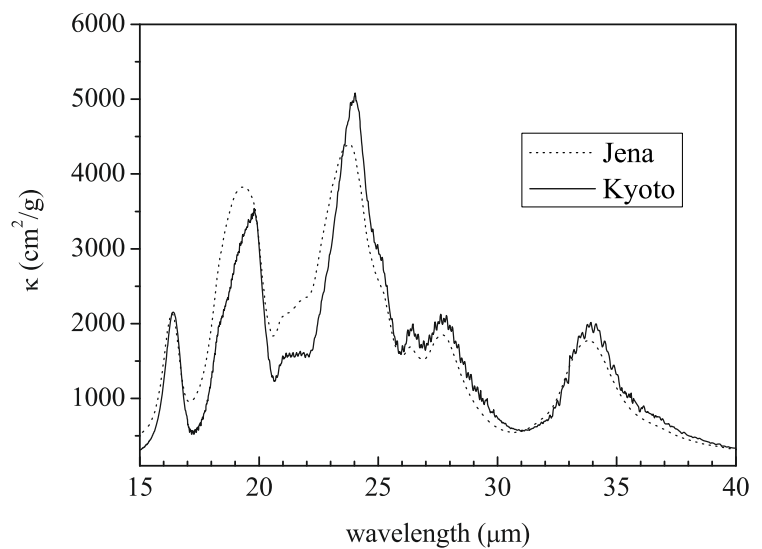

Fig. 5. IR spectra of San Carlos olivine measured by Koike et al. (2006) (Kyoto sample) and Fabian et al. (2001) (Jena sample).

was prepared by ball-milling. Consequently, it is highly probable that the difference in the spectra from different laboratories is due to differences in shape and lattice distortion of particles, caused by the different sample preparation methods: the Kyoto samples are irregular particles with less crystal lattice distortion, while the Jena samples are rounded particles with large lattice distortion. Therefore, we have to consider the difference in the sample properties when laboratory data are compared with observation data.

\subsection{Implications for astronomical observations}

The effect of crystal lattice distortion, when spectral features become broader with increasing distortion, was reported for the first time in the present experiments. Here we will discuss some implications of this effect for astronomical observations.

In the Deep Impact mission, emission features of ejecta of comet Tempel 1 were observed with the Spitzer Space Telescope, and features due to crystalline silicates were found (Lisse et al. 2006). The $33 \mu \mathrm{m}$ emission feature of Tempel 1 ejecta almost fitted that of forsterite. However, it is clear that the $33 \mu \mathrm{m}$ feature of the observation is broader than the model fit. It is suggested that this broadening is due to lattice distortion caused by impacts with hypervelocity onto the nucleus of Tempel 1

It is known that peak positions and FWHMs of peaks of IR spectra change depending on temperature and chemical composition (e.g., Koike et al. 2003; Chihara et al. 2001, 2002). In terms of temperature dependency, peak positions shift to shorter wavelength, and FWHMs decrease drastically as temperature decreases (e.g., Chihara et al. 2001; Bowey et al. 2001; Koike et al. 2006). Since we carried out all measurements of IR spectra at room temperature in this work, the variations of the FWHMs in our work are not caused by the temperature dependency. On the other hand, in terms of compositional dependence, peak positions shift to longer wavelength, and FWHMs increase with increasing iron content of Mg-rich silicates (Chihara et al. 2002; Koike et al. 2003). With observational studies, Bowey et al. (2002) and Molster et al. (2002) discussed estimation of the Fe content of olivine and temperatures of dust from the relation between the peak position and FWHM of the $69 \mu \mathrm{m}$ feature for observations of circumstellar dust.
Koike et al. (2006) superimposed temperature-dependent spectral data of forsterite on this correlation between the peak positions and $F W H M$ of the $69 \mu \mathrm{m}$ feature, and found that much observational data have lager FWHMs than the laboratory trend of the Kyoto sample, and some FWHM of observations are similar to the laboratory trend of the Jena sample. Koike et al. (2006) presented a correlation between the peak positions and FWHM using spectra of forsterite (the $\mathrm{Mg}$ end-member); the trend of the correlation shows a lower limit of peak position and an upper limit of $F W H M$ at each temperature. However, some observational data are located in the left region of the correlation trend of forsterite. These observational data means that the peak positions and the FWHMs are lower and larger than those of forsterite (=Kyoto sample). Therefore, the observational data cannot be explained only by composition and temperature effects. In contrast, as our results show, lattice distortion affects only broadening of the FWHM and does not change peak positions. Therefore, the lattice distortion effect can explain the difference between observational spectra and laboratory measurements. Consequently, circumstellar forsterite might have a lattice distortion, although the detailed conditions of circumstellar environments are not yet well known. The crystal lattice distortion might be caused by rapid growth of forsterite crystals in largely saturated conditions from either amorphous silicates or gas itself.

Acknowledgements. We would like to thank the referees for their constructive and helpful reports, which have improved this paper. This work was financially supported by JSPS Research Fellowships for Young Scientist, Grant-in-Aid from the Japanese Ministry of Education, Culture, Sports, Science, and Technology (18540243; 19104012) and Grant-in-Aid for Scientific Research on Priority Areas, "Development of Extra-Solar Planetary Science" (19015006).

\section{References}

Bohren, C. F., \& Human, D. R. 1983, Absorption and Scattering of Light by Small Particles (New York: Wiley and Sons Ins.)

Bowey, J. E., Lee, C., Tucker, C., \& Hofmeister, A. M. 2001, MNRAS, 325, 886 Chihara, H., Koike, C., Tsuchiyama, A., Tachabana S., \& Sakamoto D. 2002, A\&A, 391, 267

Fabian, D., Jäger, C., Henning, T., Dorschner, J., \& Mutschke, H. 2000, A\&A, 364,282

Fabian, D., Henning, T., Jäger, C., Mutschke, H., Dorschner, J., \& Wehrhan O. 2001, A\&A, 378, 228

Font, J., Muntasell, J., \& Cesari, E. 1997, Mater. Res. Bull., 32, 12, 1691

Jäger, C., Molster, F. J., Dorschner, J., Mutschke, H., \& Water, L. B. F. M. 1998, A\&A, 339, 940

Koike, C., Tsuchiyama, A., Suto, H., et al. 1999, in Proc. of the 32nd ISAS Lunar and Planetary Symposium, 32, 175

Koike, C., Chihara, H., Tsuchiyama, A., et al. 2000, in Proc. of the 33rd ISAS Lunar and Planetary Symposium, 33, 95

Koike, C., Chihara, H., Tsuchiyama, A., Suto, H., Sogawa, H., \& Okuda H. 2003, A\&A, 399, 1101

Koike, C., Mutschke, H., Suto, H., Naoi T., H., Chihara, et al. 2006, A\&A, 449, 583

Lisse, C. M., VanCleve, J., Adams, A. C., et al. 2006. Science, 313, 635

Molster, F. J., Waters, L. B. F. M., Tielens, A. G. G. M., Koike, C., \& Chihara, H. 2002. A\&A, 382, 241

Murata, K., Chihara, H., Tsuchiyama, A., Koike, C., \& Takakura, T. 2007, ApJ, 668,285

Ossenkopf, V., Henning, Th., \& Marthis, J. S. 1992, A\&A, 261, 567

Scherrer, P. 1918, Nachr. Ges. Wiss. Gottingen, 26 September, 98

Sogawa, H., Koike, C., Chihara, H., et al. 2006, A\&A, 451, 357

Tamanai, A., Mutschke, H., Blum, J., \& Meeus, G. 2006, ApJ, 648, L147

Waelkens, C., Waters, L. B. F. M., de Grauw, M. S., et al. 1996, A\&A, 315, L245

Waters L. B. F. M., Molster F.J., de Jong, T., et al. 1996. A\&A, 315, L361

Willson, A. J. C. 1962, X-Ray Optics (New York: Wiley and Sons Ins.) 